\title{
Prevenção do suicídio: contribuições da psicologia no âmbito da saúde pública
}

\section{Prevention of suicide: psychology's contributions to public health}

\author{
Maria Juliana da Silva Santos' ${ }^{1}$ Eduardo Mendes Medeiros ${ }^{2}$ \\ ${ }^{1}$ Autora para correspondência. Universidade das Américas. Aracati, Ceará, Brasil. ORCID: 0000-0003-4899-3745. julianaa_sillva@hotmail.com \\ ${ }^{2}$ Faculdade Maurício de Nassau. Fortaleza, Ceará, Brasil. ORCID: 0000-0003-0453-7430. eduardopsicologia88@gmail.com
}

\begin{abstract}
RESUMO | Este artigo revisou as contribuições da atuação do psicólogo na prevenção do comportamento suicida no âmbito da saúde pública. Trata-se de uma pesquisa qualitativa de revisão de literatura que utilizou a combinação dos descritores: “Prevenção do Suicídio, comportamento suicida, epidemiologia do suicídio, fatores de risco para o suicídio, fatores de proteção, atuação do psicólogo e saúde pública", nas bases de dados: Scientific Eletronic Library Online (SciELO) e na Literatura Latino-Americana e do Caribe em Ciências da Saúde (LILACS). Foram selecionadas 26 publicações que atendem ao critério de inclusão. Discutiu-se as contribuições da atuação do psicólogo na saúde pública e suas funções no contexto do SUS. Perceberam-se os desafios da atuação da equipe de saúde diante da demanda do suicídio. Não foi encontrado no levantamento das publicações nenhuma pesquisa que tratasse da atuação exclusiva do psicólogo na prevenção do suicídio. Concluiu-se que existe a necessidade de se realizarem estudos empíricos documentando os benefícios da atuação do psicólogo na prevenção do suicídio no âmbito da saúde pública para incrementar o progresso das políticas públicas de saúde.
\end{abstract}

PALAVRAS-CHAVE: Psicologia da saúde. Suicídio. Prevenção.

\begin{abstract}
This article reviews the contributions of the psychologist's role in preventing suicidal behavior in the public health context. It is a qualitative research of systematic literature review, which using the combination of the descriptors: "Suicide prevention, suicide behavior, suicide epidemiology, suicide risk factors, protection factors, psychologist performance. The following databases were consulted: Scientific Online Library in Electronic Health (SciELO) and Health Sciences Literature of Latin America and the Caribbean (LILACS). We selected 26 publications that met the inclusion criteria. The contributions of the psychologist's role in public health and their functions in the context of the SUS were discussed. The challenges of the health team's performance in the face of suicide demand were perceived. No research was found in the survey of the publications that dealt with the psychologist's exclusive role in suicide prevention. It was concluded that there is a need to conduct empirical studies documenting the benefits of the psychologist's role in preventing public health suicide to increase the progress of public health policies.
\end{abstract}

KEYWORDS: Psychology of health. Suicide. Prevention. 


\section{Introdução}

Este artigo teve como objetivo principal revisar as contribuições da atuação do psicólogo na prevenção do comportamento suicida no âmbito da saúde pública. A motivação para o desenvolvimento deste tema veio a partir da observação realizada no ano de 2013 pelo Conselho Federal de Psicologia (CFP) de que existe uma carência de publicações que tratem da importância da atuação do profissional de psicologia na prevenção do suicídio (Conselho Federal de Psicologia, 2013). Este fato é preocupante, pois vivemos em um contexto de crescente aumento de casos de suicídio tanto em nível nacional quanto em nível mundial (Bachmann, 2018; Bertolote \& Fleischmann, 2015; Freitas et al., 2013; Júnior, 2015; Machado \& Santos, 2015; Marín-León, Oliveira, \& Botega, 2012; Silva, Prates, Cardoso, \& Rosas, 2018; Teti et al., 2014).

Segundo a Organização Mundial da Saúde (OMS), o suicídio é responsável anualmente por cerca de um milhão de óbitos, sendo considerado um grave problema de saúde pública, visto que tem contribuído com mais de $2 \%$ para a carga global de doença até o ano 2020. Porém, este número não considera o impacto que o suicídio ocasiona nas vidas dos indivíduos, familiares e sociedade (Bachmann, 2018; Organização Mundial da Saúde, 2012; Organização Pan-Americana da Saúde, 2016).

Assim, a realização deste trabalho é relevante, pois traz à tona um grave problema de saúde pública que se articula com múltiplas dimensões do sujeito que exige das Políticas Públicas de Saúde o planejamento e o desenvolvimento de ações que assegurem o apoio à vida dos usuários da rede pública de saúde. É plausível, já que possibilita que se faça uma reflexão crítica sobre a real situação dos casos de suicídio entre a população brasileira. Contribui, apresentando as principais intervenções que o psicólogo desenvolve na saúde pública perante o comportamento suicida. Assim, para cumprir com o objetivo proposto, efetuou-se um levantamento de artigos e trabalhos acadêmicos que abordam questões pertinentes por meio da revisão bibliográfico-documental da literatura (Appolinário, 2009; Galvão \& Pereira, 2014; Koller, Couto \& Hohendorff, 2014).

\section{Características epidemiológicas do suicídio}

O suicídio tem se tornado um tema bastante debatido, levando o Conselho Federal de Psicologia a lançar em 2013 um livro intitulado: "Suicídio e os desafios para a Psicologia", que nasceu por meio de vários diálogos sobre o tema, onde em uma dessas discussões, a historicidade do termo foi explanada demonstrando que o termo suicídio surgiu por volta do século XII. Anteriormente, o que existia eram formas de se relatar o ato. Porém, foi por meio de Santo Agostinho que o suicídio passou a ter um sentido condenável. E na Idade Média, o suicídio era entendido como crime, haja vista que prejudicava os negócios da Coroa. Já no fim da Idade Média ocorreu a divisão entre a Coroa e a Igreja, assim abriu-se um espaço para o domínio do conhecimento médico, que exerceu importante influência sobre a sociedade. Dessa forma, os médicos passaram a desvincular a morte voluntária do pecado e classificando-a como loucura (Conselho Federal de Psicologia, 2013).

Contudo, na literatura existem alguns autores que procuraram estudar o suicídio e dentre eles encontra-se o sociólogo francês Émile Durkheim, que publicou sua obra intitulada "Le Suicide" (O Suicídio), afirmando que o suicídio é todo ato de morte cometido pelo próprio sujeito. Já a tentativa de suicídio foi definida como uma ação deliberada, mas interrompida antes que cause a morte. Durkheim apresenta em seu livro o suicídio como um fenômeno puramente social, e não individual, enfatizando que é uma doença da época, onde salienta a grande influência que a sociedade desempenha nos sujeitos, inclusive quando se trata da ordem pessoal, isto é, a decisão de permanecer vivo ou escolher a própria morte (Durkheim, 2014).

Além disso, o mesmo autor em seus escritos classifica que há três tipos de suicídio, conforme o nível de integração social do sujeito. A saber, o egoísta, o altruísta e o anômico. Sendo que o primeiro tipo é o suicídio egoísta, no qual o indivíduo não está profundamente interligado a um grupo social, tendo como fatores a depressão, a melancolia e a sensação de desamparo moral que é atribuído por separação do grupo. O segundo tipo é o suicídio altruísta, onde o sujeito sacrifica-se em benefício do grupo, sendo compreendida como uma obrigação e se não for obedecida o sujeito 
é penalizado pela desonra e por punições religiosas. E por último, o terceiro tipo é denominado de anômico e ocorre em indivíduos que vivem em uma sociedade em crise, onde as regras não existem ou deixaram de ser importantes (Durkheim, 2014).

Já o termo comportamento suicida abrange tanto a tentativa de suicídio, a ideação suicida sem tentativas, o risco de suicídio e o planejamento e consumação do ato. Ou seja, as condutas suicidas são caracterizadas como um processo difícil, visto que pode divergir desde a ideia de retirar a própria vida, que pode ser notificada por meios verbais e não verbais, até projetar a ação, a tentativa e a execução do ato (Conselho Federal de Psicologia, 2013; Kohlrausch, 2012; Organização Pan-Americana da Saúde, 2016).

Quanto às características epidemiológicas, as faixas etárias que apresentam taxas de suicídio mais baixas são em indivíduos menores de 15 anos. E as maiores taxas encontram-se entre os sujeitos acima de 70 anos, em ambos os sexos, praticamente em todas as regiões do mundo. Assim, um estudo realizado por Wasserman, Cheng e Jiang (2008) sobre os índices de suicídio entre jovens, com dados de 90 países, avaliou em 7,4/100 mil a taxa entre jovens de 15 e 19 anos. Onde apresentou as maiores taxas em países como: Sri Lanka $(46,5 / 100$ mil), Lituânia (23,9/100 mil) e Rússia (23,6/100 mil). No Brasil, o índice foi de 4,2/100 mil, sendo considerado baixo em escala mundial, ocupando a $71^{\circ}$ posição. Também foram averiguados os maiores percentuais entre jovens do sexo masculino $(10,5 / 100 \mathrm{mil})$ do que do sexo feminino (4,1/100 mil) (Júnior, 2015; Waiselfisz, 2014; Wasserman, Cheng \& Jiang, 2008).

No ano de 2012, a Organização Mundial da Saúde (OMS) analisou os números mundiais de suicídio que foram de 11,4 mortes por 100 mil habitantes. $\mathrm{Na}$ qual os maiores percentuais localizam-se no Leste Europeu e as mais baixas na América Latina e na Europa Ocidental. Enquanto Estados Unidos e Oceania apresentam taxas intermediárias. Já os dados dos países africanos não são fidedignos, impedindo os números exatos das taxas. Assim, em números totais, os países com mais mortes por suicídio são a Índia, China, Estados Unidos, Rússia, Japão, Coréia do Sul, Paquistão e Brasil. Entretanto, os países que se destacam em índices de suicídio são Guiana, Coréia do Sul, Coréia do Norte, Sirilanka, Lituânia, Suriname, Moçambique e Nepal. Logo, os países populosos como a China, Índia e Brasil, embora possuam elevadas taxas de ocorrências, expõem baixos percentuais de suicídios. Mas a Guiana, com menos de um milhão de moradores, mostra a maior taxa mundial com 44,0/100 mil habitantes (Júnior, 2015; Waiselfisz, 2014).

Dessa forma, em uma pesquisa realizada para averiguar os números de suicídio no Brasil, entre 1980 a 2012, foi constatado que a soma por ano elevou-se de $3.896 \mathrm{ca}$ sos para 10.321 , um acréscimo de $62,5 \%$. E também foi observado no estudo que entre os anos de 2002 a 2012 houve um acréscimo de $33,6 \%$, maior que o aumento do número de homicídios (2,1\%), de mortes por acidentes de veículos (24,5\%) e do aumento da população do Brasil no mesmo período (Waiselfis, 2014).

Ainda segundo o mesmo autor, a distribuição das taxas de suicídio em relação ao sexo no Brasil acompanha a tendência mundial, em que os homens praticam mais suicídio que as mulheres. Sendo assim, é mais comum entre os homens e a tentativa de tirar a própria vida é mais frequente entre as mulheres. Dessa forma, os índices de suicídio entre os homens têm uma tendência a subir, enquanto que entre as mulheres a diminuir. Também entre o período de 1980 a 2012 houve um crescimento nas mortes por suicídio em todas as faixas etárias, menos entre as mulheres jovens. Porém, o maior acréscimo foi entre homens jovens, indo de 5,7 em 1980 para 8,9 em 2012, aumento de 54,1\%.

Embora os índices tenham crescido no Brasil, encontram-se variações entre as regiões brasileiras. O maior índice está na região Sul, com 9,8/100 mil habitantes e no Centro-Oeste, com 7,6/100 mil, seguidas pelo Sudeste com 5,6, a região Norte com 5,3 e o Nordeste com 5,2. Ainda que as regiões Sul e Centro-Oeste possuam os maiores índices entre o período de 2000 a 2012, as mortes por suicídio têm baixado nessas duas regiões, porém, ocorreu um aumento nos índices das demais. Entre 2002 a 2012, na região Norte, as mortes por suicídio subiram $37,2 \%$, indo de 3,8 para 5,3/100 mil habitantes. O Nordeste teve o maior acréscimo no índice nos últimos 13 anos, crescimento de 72,4\%, indo de 3,0 em 2000 para 5,2/100 mil habitantes em 2012. No Sudeste, os óbitos por suicídio cresceram $34,8 \%$, saindo de 4,2 em 2000 para 5,6 em 2011. E no Centro-Oeste ocorreu à maior queda nacional de 2000 para 2011, mais de 13\% e de 2011 para 2012 decresceram 1,8\% (Machado \& Santos, 2015). 
Da mesma forma, a pesquisa de Waiselfisz (2014) mostra quais estados brasileiros apresentam as mais altas taxas de mortes por suicídio e as menores taxas no ano de 2012. Logo, o Rio Grande do Sul aponta o índice de 10,9; Santa Catarina com 8,6; Mato Grosso do Sul com 8,4 e Roraima com 8,1. Esses estados lideraram índices mais altos do país. Porém, as unidades da Federação que expõem as taxas mais baixas são Rio de Janeiro com 2,9, Amapá com 3,0 e Pará 3 , 1. A pesquisa também expõe os números de suicídio nos estados do Nordeste no ano de 2012, onde o Piauí apresentou a taxa de 7,4 e Ceará 5,9 por 100 mil habitantes. São os maiores índices de mortes por suicídio, acima da média nacional. E o Rio Grande do Norte apresentou a taxa de 5,3 mortes por 100 mil habitantes, seguido do estado de Sergipe com 5,1 por 100 mil habitantes. A Paraíba encontra-se na quinta colocação, com uma taxa de 4,9 suicídios por 100 mil habitantes e o estado de Pernambuco com um índice de 3,8 por 100 mil habitantes. Assim, o Maranhão configura-se com a menor taxa com 3,1 por 100 mil habitantes, seguido pela Bahia e Alagoas, ambos com 3,4 por 100 mil habitantes (Waiselfisz, 2014).

Em relação aos métodos utilizados para o suicídio, algumas pesquisas apontam que em países de alta renda, os principais meios para o ato são os enforcamentos, empregados em $50 \%$ dos casos, e o uso de armas de fogo com $18 \%$. Nas zonas rurais e em países de baixa ou média renda, o uso de pesticida se destaca, pois representa 30\% das ocorrências de suicídio no mundo. E os métodos mais usados no Brasil são enforcamento, lesão por armas de fogo e autointoxicação por pesticidas, correspondendo em torno de $80 \%$ das ocorrências (Machado \& Santos, 2015).

No entanto, mesmo com a repercussão acerca do alto índice de casos, interessa salientar que estes números não expõem com exatidão a realidade, visto que os índices de suicídio são ainda maiores do que os revelados. Tendo em vista as subnotificações (Trigueiro, 2015).

\section{Principiais fatores de riscos e de proteção para o suicídio}

Segundo a literatura de Carvalho et al. (2013), fatores de risco são contextos, fatos de vida, patologias ou traços de personalidade que são capazes de intensificar as possibilidades de alguém tentar o suicídio ou suicidar-se. Ainda conforme os mesmos autores, o conhecimento dos fatores de risco e de proteção fazem-se indispensáveis em uma estratégia de prevenção do suicídio, haja vista que colabora para identificar a natureza e o tipo de intervenção adequada, sendo sugestivo do contexto em que o sujeito se encontra vulnerável para o ato.

Dessa forma, quando se localizam uma sucessão de fatores negativos ocorre uma maior possibilidade de comportamentos suicidas. Assim como facilita avaliar o nível geral do risco de suicídio para uma pessoa e coopera para o andamento dos programas de tratamento que se referem aos diversos fatores implicados, fatores esses que podem ser identificados e alterados.

Carvalho et al. (2013) ressaltam que não existe um único fator de risco ou protetor que provoque ou impeça o comportamento suicida, haja vista que nem todos os fatores são igualmente relevantes em condições de prevenção. Diante disso, precisam ser avaliados em conjunto e inseridos no contexto do sujeito e de sua historicidade. Assim, são muitos os fatores que influenciam no comportamento suicida, sendo que um único fator não é satisfatório para esclarecê-lo. Geralmente, vários fatores vão somando-se e deste modo aumenta a vulnerabilidade do sujeito em apresentar o comportamento suicida. Logo, podendo estar associado a múltiplos fatores como psicológicos, econômicos, sociais e culturais.

Quanto aos possíveis riscos, estudos revelam alguns fatores sociodemográficos, como homens, grupos etários entre 15 e 35 anos ou acima de 75 anos, situações econômicas extremas, pessoas muito ricas ou muito pobres, moradores em áreas urbanas, desempregados, principalmente aqueles que perderam recentemente o emprego, aposentados, ateus, solteiros ou divorciados e migrantes. E os fatores socioculturais, como o afastamento social, a falta de apoio social, o preconceito relacionado ao comportamento de buscar ajuda. Assim como obstáculos ao acesso aos serviços de saúde - particularmente saúde mental e tratamento do uso de substâncias, crenças culturais e religiosas em relação ao suicídio, tendo o ato como uma saída digna de um conflito individual. Tal como a influência de outros comportamentos suicidas, inclusive vinculados pela mídia. Por último, os fatores situacionais, como os métodos disponíveis para praticar, contexto familiar conturbado - como abuso físico ou sexual e negligência familiar. Bem como, histórico dessa atitude na família ou de transtorno mental (Organização Mundial da Saúde, 2012; Carvalho et al., 2013). 
Contudo, em outra pesquisa realizada no Hospital Universitário de Santa Catarina, buscaram identificar a influência das doenças sobre o comportamento suicida, no qual foi avaliada a ideação dos pacientes hospitalizados em diferentes especialidades como gastroenterologia, clínica médica, cardiologia, pneumologia, hematologia, neurologia e endocrinologia. Os resultados evidenciaram a prevalência de ideação suicida de $7,2 \%$, número maior ao achado na população em geral (Stefanello \& Furlanetto, 2012). Dessa forma, a doença física pode intensificar o risco, principalmente se estiver relacionado a déficits funcionais, deformação da imagem corporal, dor crônica, ser dependente de outras pessoas. As enfermidades de maior risco são as doenças neurológicas, oncológicas e pulmonar obstrutiva crônica (Organização Mundial da Saúde, 2012; Carvalho et al., 2013).

Além disso, dentre as causas de risco ressalta-se a tentativa prévia de suicídio, que constitui o fator de risco mais relevante. Visto que pacientes que tentaram se suicidar previamente têm de cinco a seis vezes mais probabilidade de tentar outra vez. Assim, verifica- se que $50 \%$ daqueles que se suicidaram já haviam tentado antes (Conselho Federal de Medicina, 2014; Ferreira, 2014; Trigueiro, 2015).

Os aspectos psicológicos também são fatores de risco para o comportamento suicida, onde se inclui o estado psíquico em que o sujeito mostra uma dor emocional intolerável e agonia por não poder aliviar o sofrimento pelo qual está vivenciando. Já a impulsividade e agressividade caracterizam-se como fatores de risco, sobretudo entre adolescentes e jovens (Organização Mundial da Saúde, 2012; Conselho Federal de Medicina, 2014).

Ainda, as vulnerabilidades da personalidade, como a capacidade para conduzir a dor psicológica, os traços de personalidade e as habilidades para solucionar dificuldades usando os recursos internos e externos são fatores relevantes que podem apaziguar ou aumentar o risco de suicídio. Os traços de personalidade regularmente relacionados ao risco são o desamparo, hostilidade, rigidez, perfeccionismo e dependência. Assim, os altos níveis de desesperança com ou sem depressão estão ligados a um elevado fator de risco (Carvalho et al., 2013).
Também a presença de transtorno mental é uma causa de risco que está mais estreitamente ligada ao suicídio, independente da faixa etária, tendo como os principais: os transtornos de humor (depressão e transtorno bipolar), dependência de álcool e outras substâncias, esquizofrenia e transtornos de personalidade como borderline (Miranda, 2014).

Assim como, existem fatores de risco para o suicídio, há também os de proteção. Onde os protetores equivalem às características individuais e contextos coletivos e socioculturais, e quando presentes ou reforçados, estão ligados à prevenção dos comportamentos suicidas. Assim, os fatores protetores individuais são: a habilidade para solucionar problemas e conflitos, disposição para pedir ajuda, concepção de valor pessoal, estar aberto para novos conhecimentos, recursos comunicativos desenvolvidos, dedicação aos projetos de vida. E as questões familiares, como o bom convívio familiar, ajuda da família, vínculos de confiança. Por último, as questões sociais: estar empregado, oportunidade de fazer uso dos serviços de saúde, os valores culturais, pertencer a uma religião (Organização Mundial da Saúde, 2012; Conselho Federal de Medicina, 2014; Júnior, 2015).

Além disso, algumas questões como segurança em geral, alimentação apropriada, períodos adequados de repouso, relações afetivas, incluir-se na comunidade, possuir um parceiro fixo e filhos ainda dependentes podem colaborar para a estabilização da saúde física e mental, bem como diminuir os efeitos dos fatores de riscos ou transtornos mentais (Ferreira, 2014).

A maior parte das ocorrências de autoagressão é atendida em algum tipo de serviço de saúde, que disponibiliza assistência aos pacientes que tentam o suicídio. Todavia, a instituição de saúde apropriada para os primeiros atendimentos é o hospital geral de emergência, visto que oferece suporte de técnicos e equipe preparada para dar assistência às pessoas em risco de morte (Gondim, 2015). Sabendo-se que a ingestão de pesticidas e de medicamentos são formas regularmente usadas em tentativas de suicídios, a procura dessas unidades é grande. Sendo assim, esses pacientes chegam encaminhados por vários locais, como Unidades de Pronto Atendimento (UPA), Centros de Saúde, pelo Serviço de Atendimento Móvel de Urgência (SAMU), por bombeiros ou pela procura espontânea da população (MacChiaverni, Borges \& Oliveira, 2013). 
Além disso, a emergência é uma unidade de saúde que dispõe de algumas características como a alta rotatividade de pacientes e a curta permanência dos usuários no serviço, requerendo do profissional de psicologia que desempenhe um atendimento psicoterápico breve e focal. Logo, a unidade de emergência hospitalar representa um papel fundamental na prevenção do suicídio, pois, geralmente, é o primeiro serviço de saúde que o sujeito ou família busca depois da tentativa de suicídio. Neste espaço, iniciam-se as intervenções com o propósito de reduzir o sofrimento do paciente, mas, posteriormente, essas intervenções devem ter seguimento em outros serviços de saúde (Ferreira \& Gabarra, 2014; Gonçalves, Silva, \& Ferreira, 2015; Gondim, 2015; Organização PanAmericana da Saúde, 2016).

Assim, quando o paciente recebe assistência nos serviços de saúde depois da tentativa, a avaliação de risco deve acontecer desde o primeiro momento e durante a sua internação no hospital. Esse primeiro contato com o usuário do serviço é uma ótima oportunidade para que os profissionais verifiquem o nível de risco e possam intervir para diminuí-lo. Sendo assim, a relação terapêutica constitui-se como um instrumento relevante para aumentar a adesão e alcançar resultados significativos no acolhimento. Pois desenvolver uma boa relação como o paciente pode gerar uma ótima impressão sobre a qualidade da assistência ofertada e auxilia na prevenção de novas tentativas (Vidal \& Gontijo, 2013).

No entanto, conforme Gonçalves, Silva e Ferreira (2015), Gondim (2015), Vidal \& Gontijo (2013) e Zana e Kovács (2013), nem sempre a equipe sabe aproveitar essa oportunidade, seja pelas particularidades do serviço ou pela falta de formação adequada para atender pacientes suicidas. Esse despreparo dos profissionais pode ocasionar posições e condutas inadequadas, comprometendo o processo terapêutico. Isso pode manifestar dificuldades ao tratar acerca do tema dentro da equipe de saúde e no contato com o próprio paciente ou seus familiares. Essa conduta prejudica a atuação profissional, onde se pode relacionar-se à incompetência para auxiliar o paciente em seu sofrimento.

Contudo, o psicólogo, por fazer parte de cada setor do hospital, oferece assistência ao paciente, prestando orientações e acompanhamento aos familiares e a equipe. A sua principal função é avaliar e conduzir intercorrências psicológicas dos usuários que estão em acompanhamento no hospital. Sendo assim, na emergência, é habitual ocorrer dificuldades na assistência aos usuários do serviço. Em geral, a equipe não oferece uma atenção adequada aos pacientes, pois muitas vezes não considera a tentativa de suicídio como uma expressão de um transtorno mental que pode levar à morte. Onde é habitual o profissional de saúde, diante do comportamento suicida, constituir seu julgamento fundamentado em suas próprias convicções, podendo ser intolerante, indiferente e dar lições de ânimo ou de religiosidade. E raramente o profissional de saúde procura ouvir o paciente ou se esforça para conhecer sem julgamentos o que levou a tentativa de suicídio (Gondim, 2015).

Ainda segundo o mesmo autor, o psicólogo, por fazer parte da equipe de saúde mental, configura-se como o principal agente de transformação nessas ocorrências, visto que a sua atuação abrange o acoIhimento, a escuta, o atendimento, as orientações e encaminhamento às unidades da rede de saúde mental. Sendo assim, a função do psicólogo também abrange intervenções junto a equipe de saúde, no sentido de ajudar nas relações entre à equipe e a família. Também, nesse ambiente, esse profissional atua desde o primeiro momento que o paciente chega à unidade, ou após os procedimentos iniciais necessários. Porém, quando na emergência chega um paciente que não tem condições de se fazer um acoIhimento inicial, pois se encontra em estado grave, o profissional primeiramente inicia suas atividades acolhendo a família até o paciente ter condições de ser atendido. E, depois da alta médica, o psicólogo é o encarregado para realizar o encaminhamento ou transferência ao serviço de referência para tentativas de suicídio. Assim, tornando-se o profissional de referência tanto para a assistência integral quanto para o paciente durante a sua internação.

Nesse sentido, ainda segundo o mesmo autor, o profissional da psicologia, frente ao comportamento suicida, deverá analisar o nível de sofrimento do paciente, verificando se ele tem desejo de viver. Assim como, quando estiver a escutar o sujeito, poderá ajudá-lo a enxergar outros caminhos para sua própria vida. $E$, no contexto do acolhimento e acompanhamento, deve proporcionar um espaço para que o paciente fale de sua angústia, tristeza e dor, oferecendo-Ihe uma escuta sem julgamentos. Bem como deve 
criar um contrato de não suicídio, isso quando o sujeito precisa de um ponto de apoio e o vínculo entre profissional e paciente já está formado. E, por último, expandir o cuidado para a família e redes de apoio que o paciente está inserido. Nessa perspectiva, vamos abordar outro estudo voltado para a elaboração de um instrumento de registro de atendimento psicológico aos pacientes por tentativa de suicídio, no Hospital da Universidade Federal de Santa Catarina. Os autores assinalam que é importante compreender o instrumento de registro, além das informações técnicas, pois se deve acolher o sofrimento do paciente, oferecendo uma escuta ao que é verbalizado. E também se deve apontar, junto ao paciente, o que proporciona o desejo de viver. Oferecendo-lhe esperança para manter seu desejo de permanecer vivo (MacChiaverni, Borges \& Oliveira, 2013).

Ademais, dentro da saúde pública além do contexto hospitalar, o psicólogo atua também na prevenção do suicídio nos Centros de Atenção Psicossocial (CAPS), onde segundo a Associação Brasileira de Psiquiatria, são serviços secundários do Sistema Único de Saúde assegurando que todos os sujeitos depois de uma tentativa de suicídio sejam assistidos nas primeiras setenta e duas horas após a tentativa por, no mínimo, um médico psiquiatra (ABP, 2014). Sendo assim, os CAPS se caracterizam como os principais dispositivos de saúde mental e se constituem em vários tipos como os CAPS I, II e III que são para os transtornos mentais severos e persistentes. Já os CAPS AD ॥ e III, destinam-se para o tratamento de sujeitos com transtornos decorrentes do uso de álcool e outras drogas e os CAPS I para crianças e adolescentes com transtornos mentais severos (Madeira, 2014).

Em um estudo com a finalidade de apresentar a atuação dos profissionais de um CAPS diante de usuários com tentativa e risco de suicídio, destacou-se o acolhimento como um instrumento que favorece o atendimento humanizado e também o trabalho interdisciplinar realizado pelos profissionais. Onde foi assinalada a importância do trabalho entre o médico, a enfermeira, a psicóloga e a assistente social, tendo o trabalho em equipe como uma estrutura para responder as ocorrências de risco de suicídio existentes na comunidade. Assim, na pesquisa os profissionais citaram um elemento de fundamental relevância que é a formação ou resgate do vínculo, com o paciente e com a sua família, nas circunstâncias de crises (Heck et al., 2012).
Dessa maneira, segundo as ideias dos mesmos autores 0 atendimento necessita ser eficiente, dialogal e consciente, a fim de intervir e oferecer assistência de maneira humana e empática para o sujeito que apresenta o comportamento suicida. Nesse aspecto, a família se caracteriza como uma aliada para ajudar os profissionais a entenderem o que induziu o sujeito a tentar o suicídio e a superar as ocasiões de sofrimento psíquico. Assim, a aproximação familiar do sujeito em sofrimento também colabora para desmistificar algumas crenças formadas pelo paciente, como se a morte fosse extinguir todas as dificuldades e conflitos existentes. Logo, quando essas motivações são ouvidas ocorre uma maior probabilidade de impedir o comportamento suicida, haja vista que, muitas vezes, a família busca auxílio nos dispositivos de saúde da comunidade.

Também após a internação hospitalar a família pode cooperar para a prevenção dos comportamentos suicidas. Onde os profissionais de saúde podem realizar intervenções, como orientar a família a organizar a rotina do paciente para evitar o acesso a utensílios que venham a ser usados para cometer o suicídio. Além disso, buscar ouvir esse familiar quanto às suas preocupações, inclusive como lidar com a pessoa que cometeu autoagressão. Outras intervenções como atendimentos psicoterápicos individuais ou grupais são estratégias usadas pelos profissionais para ajudar esses sujeitos que são atingidos pelo ato suicida, visto que os conflitos familiares podem transformar o contexto em uma situação mais doentia (Figueiredo, 2012; Oliveira et al., 2016).

Em suma, de acordo com os autores citados acima, a intervenção dos profissionais de saúde não necessita ser realizada exclusivamente no hospital. Pois o trabaIho em rede necessita ser continuamente estimulado para que ocorra uma comunicação entre os vários dispositivos de saúde, como o hospital, unidades básicas de saúde, CAPS, dentre outros. Dessa forma, é capaz de se eliminar o trabalho fragmentado, operando ao mesmo tempo sobre os fatores de prevenção e promoção em saúde mental (Gonçalves, Silva, \& Ferreira, 2015; Gondim, 2015; Organização Pan-Americana da Saúde, 2016; Zana \& Kovács, 2013). 


\section{Método}

Este trabalho trata-se de uma pesquisa qualitativa que se justifica, pois a análise qualitativa torna possível a atuação do pesquisador na pratica profissional, e de pesquisa, decompondo a subjetividade dos fenômenos em processo de investigação, suscitando dados que dão fundamento às evidências dos objetivos propostos pela pesquisa (Campos, 2008).

Deste modo, foi realizada uma revisão da literatura, que é um método que permite maximizar o potencial de uma busca, encontrando o maior número possível de resultados de uma maneira organizada fazendo uma análise crítica das publicações, tendo como finalidade sistematizar, compor e julgar pesquisas importantes sobre um tema delimitado (Galvão \& Pereira, 2014; Koller, Couto \& Hohendorff, 2014).

Realizando também, uma pesquisa documental, que se justifica, pois o conceito de documentos abrange uma gama maior de publicações que contenham informações registradas que formem uma unidade, proporcionando suporte que possa servir para consulta, estudo ou prova (Appolinário, 2009).

\section{Procedimentos}

Para a realização desta pesquisa, foi determinada a pergunta de pesquisa que norteou a realização do estudo: Quais as contribuições da atuação do psicólogo na prevenção do comportamento suicida no âmbito da saúde pública?

Definiu-se no sistema DeCS/MeSH e Biblioteca Virtual em Saúde em Psicologia (BVS-Psi Brasil) os descritores: "Prevenção do Suicídio, comportamento suicida, epidemiologia do suicídio, fatores de risco para o suicídio, fatores de proteção do suicídio, psicólogo e saúde pública".

Definiu-se como critérios de inclusão: livros; ensaios; documentos foram limitados a cartilhas de prevenção ao suicídio; dissertação de mestrado; tese de doutorado; artigos originais e de revisão publicados no período entre os anos de 2012 a 2018, escritos em língua portuguesa, que tratam sobre o objetivo deste estudo que é revisar as contribuições da atuação do psicólogo na prevenção do comportamento suicida no âmbito da saúde pública.
Foram excluídos os estudos que não levam em consideração as contribuições da atuação do psicólogo na prevenção do comportamento suicida no âmbito da saúde pública; os estudos que contenham pouca análise conceitual dos objetos desta pesquisa; estudos que se distanciam da questão abordada; artigos publicados que se repetem e estudos com data de publicação anterior ao ano de 2012.

Posteriormente, em busca de publicações científicas em língua portuguesa, consultou-se as bases de dados eletrônicas: Scientific Eletronic Library Online (SciELO) e na Literatura Latino-Americana e do Caribe em Ciências da Saúde (LILACS) utilizando a combinação dos descritores já mencionados. A coleta de dados foi realizada nos meses de fevereiro de 2017 a março de 2018.

Foram encontrados 30 resultados, porém, após passarem por uma leitura preliminar de seus resumos a fim de verificar a eficiência e viabilidade do material coletado para a posterior análise e discussão dos materiais encontrados. E também serem submetidos aos critérios de inclusão e de exclusão foram selecionados 26 documentos que atendem aos critérios metodológicos estabelecidos. Em seguida foi realizada a leitura dos textos, para que se pudesse fazer uma reflexão atualizada sobre o tema proposto.

O presente estudo limitou-se a realizar uma pesquisa de revisão bibliográfico-documental sobre as contribuições da atuação do psicólogo na prevenção do comportamento suicida no âmbito da saúde pública, tendo em vista nossas limitações estruturais, temporais e financeiras, as limitações específicas já foram anteriormente expostas no item critérios de inclusão e exclusão.

\section{Discussão dos resultados}

Ficou evidente que ocorre uma variação das taxas de suicídio em relação à faixa etária, ao sexo e regiões geográficas. Onde o Brasil segue a tendência mundial, sendo o suicídio mais praticado pelos homens e a tentativa de suicídio, mais frequente entre as muIheres. Dessa forma, aprende-se que tal índice pode ser atribuído porque os homens empregam métodos mais letais para cometer o suicídio, são mais impulsivos e procuram menos auxílio para problemas psicológicos. Assim como possuem uma taxa maior de 
abuso de álcool e drogas ligadas à depressão e a obrigação atribuída pela sociedade de ser o mantenedor da casa (Carvalho et al., 2013, Ferreira, 2014).

Quanto às variações geográficas, o Brasil apresentou entre 2000 a 2012 os números de suicídio mais elevados na região Sul e o maior aumento percentual foi no Nordeste. Onde, no ano de 2012, os estados do Piauí e Ceará tiveram os maiores índices, acima da média nacional (Waiselfisz, 2014; Machado \& Santos, 2015).

Contudo, nas pesquisas foi evidenciado que o grupo que apresentou taxas de suicídio mais baixas foram os indivíduos menores de 15 anos. Já às faixas etárias com maior risco de suicídio estão pessoas que estão no final da adolescência e início da vida adulta e depois dos 70 anos. Assim, percebe-se que o elevado índice de suicídios entre idosos pode ser pelo isolamento social, facilidade para obter remédios e o luto. Já a maior taxa no fim da adolescência pode ser em razão dos transtornos mentais, como a depressão que começa neste período (Waiselfisz, 2014; Ferreira, 2014). Bem como a impulsividade e agressividade caracterizam-se como fatores de risco, sobretudo entre adolescentes e jovens (Organização Mundial da Saúde, 2012; Conselho Federal de Medicina, 2014).

Ressalta-se na literatura que o suicídio acontece por uma série de fatores e patologias, sendo o transtorno mental uma causa de risco que está mais estreitamente ligada, independentemente da faixa etária, sendo os principais: os transtornos de humor (depressão e transtorno bipolar), dependência de álcool e outras substâncias, esquizofrenia e transtornos de personalidade, como borderline (Miranda, 2014; Júnior, 2015). Entretanto, dentre os principais fatores de risco destaca-se a tentativa prévia de suicídio, que constitui o fator de risco mais relevante. Já que, na tentativa contínua, geralmente são empregados métodos mais letais e que oferecem maior gravidade (Ferreira, 2014; Conselho Federal de Medicina, 2014; Trigueiro, 2015).

Assim, percebeu-se que não existe um único fator de risco ou protetor que provoque ou impeça o comportamento suicida, haja vista que nem todos os fatores são igualmente relevantes em condições de prevenção. Diante disso precisam ser avaliados em conjunto e inseridos no contexto de vida do sujeito e de sua historicidade. Dessa forma, não se pode definir um fator para o suicídio e sim desencadeadores, que em alguma circunstância podem estimular o comportamento suicida (Carvalho et al., 2013). Estudos comprovam que no período da adolescência o suicídio é um fenômeno complexo e multifatorial, abrangendo fatores biológicos, psicológicos, sociodemográficos e culturais (Braga \& Dell'Aglio, 2013). Quanto aos fatores protetores, os estudos apresentam as características e circunstâncias individuais, coletivas e socioculturais que, quando presentes e reforçadas, estão ligadas à prevenção dos comportamentos suicidas (Organização Mundial da Saúde, 2012; Organização Pan-Americana da Saúde, 2016; Conselho Federal de Medicina, 2014).

\section{Atuação do psicólogo na saúde pública e suas funções no contexto do SUS}

Ademais, as pesquisas revelaram que as instituições públicas responsáveis para tratar as pessoas com tentativas de suicídio são: as Emergências dos Hospitais Gerais e os Centros de Atenção Psicossocial (CAPS). Onde os profissionais dos Hospitais Gerais e dos CAPS possuem um papel fundamental na prevenção do suicídio, já que, geralmente, os primeiros dispositivos de saúde que o sujeito ou família busca depois da tentativa do ato (Ferreira; Gabarra, 2014; Gondim, 2015; Oliveira et al., 2016).

No entanto, identificou-se que muitos profissionais da rede pública de saúde estão despreparados para o acolhimento aos pacientes que apresentam o comportamento suicida. E esse despreparo pode ocasionar posições e condutas inadequadas, comprometendo o processo terapêutico. Porém, pode-se averiguar que tais dificuldades podem ser em razão da emergência do hospital se constituir em um espaço de tensão e estresse. Também pela grande demanda do serviço, a falta de capacitação dos profissionais e as deficiências do sistema ou até mesmo por não saber administrar o tempo na emergência, levando os profissionais a atuarem de forma impessoal. (Gonçalves, Silva \& Ferreira, 2015; Vidal \& Gontijo, 2013).

Contudo, é importante assinalar que essas dificuldades dos profissionais em não saberem trabalhar com pessoas que tentaram o suicídio podem ser pela lacuna na formação desses profissionais quanto ao tema. Pois a carência de conhecimentos foi exposta pela maioria das pesquisas, onde os profissionais descreveram não ter recebido um treinamento acadêmico 
ou hospitalar para lidar com o comportamento suicida. Além disso, a capacitação deficiente pode relacionar-se à incompetência para auxiliar o paciente em seu sofrimento (Vidal \& Gontijo, 2013; Oliveira et al., 2016).

Dessa forma, é fundamental salientar que o suicídio, pode ser apontado como um tabu, onde muitos não têm o interesse em discutir acerca do tema. Mesmo com as inúmeras consequências ocasionadas pelo elevado índice de ocorrências, o tema precisa ser debatido, pois sem conhecimento a população não o percebe como um problema. Contudo, as taxas de suicídio não tem sido suficientes para mudar esse cenário. Sendo necessário romper esse paradigma, visto que não se conseguirá diminuir os dados estatísticos sem informação (Trigueiro, 2015). Assim, nota-se a importância desse tema ser discutido dentro das universidades, já que é complexo e traz consequências devastadoras na vida daqueles que expõem o comportamento suicida (Organização Pan-Americana da Saúde, 2016; Vidal \& Gontijo, 2013).

A partir disso, alguns autores fazem alguns questionamentos sobre a real situação do comportamento suicida, assim como os processos de trabalho e as relações com as Instituições de Ensino Superior. Onde afirmam que pelo fato do suicídio ser um problema de saúde pública, as Universidades necessitariam discutir mais acerca dessa temática. Como também não deveria faltar treinamento para esses profissionais atuarem de maneira apropriada diante dos casos, pois os atos suicidas se configuram como uma realidade no contexto hospitalar (Oliveira et al., 2016).

As produções científicas apresentam que o psicólogo é o principal agente de transformação nas ocorrências de suicídio. Pois a atuação do profissional envolve acoIhimento, escuta, atendimento, orientações e encaminhamento aos dispositivos da rede de saúde mental (Conselho Federal de Psicologia, 2013; Gonçalves, Silva, \& Ferreira, 2015; Gondim, 2015; Zana \& Kovács, 2013). Sendo assim, a atuação do profissional da psicologia diante do comportamento suicida também abrange intervenções junto à equipe de saúde, no sentido de facilitar nas relações entre a equipe e a família e promover a educação em saúde junto a equipe de saúde (Gondim, 2015; MacChiaverni, Borges \& Oliveira, 2013; Organização Pan-Americana da Saúde, 2016).
Assim, o trabalho do psicólogo consiste principalmente em analisar o nível de sofrimento do paciente, verificando se ele tem desejo de viver. Assim como, quando estiver a escutar o sujeito, poderá ajudá-lo a enxergar outros caminhos para sua própria vida. $\mathrm{E}$ proporcionar um espaço para que o paciente fale de suas angústias, oferecendo-lhe uma escuta sem julgamentos. Bem como criar um contrato de não suicídio, isso quando o sujeito precisa de um ponto de apoio e o vínculo entre profissional e paciente já está formado. E também deverá expandir o cuidado para com a família e redes de apoio que o paciente está inserido (MacChiaverni, Borges \& Oliveira, 2013).

\section{Desafios da atuação na equipe de saúde diante da demanda do suicídio}

Outro aspecto relevante apresentado nas pesquisas é a atuação do psicólogo junto à família do paciente. Onde os profissionais de saúde podem realizar intervenções, como orientar a família a organizar a rotina do paciente para evitar o acesso a utensílios que venham a ser usados para cometer o suicídio. Além disso, buscar ouvir esse familiar acerca de suas preocupações. Inclusive poderão realizar atendimentos psicoterápicos para ajudar esses sujeitos que são atingidos pelo ato suicida (Figueiredo, 2012; Oliveira et al., 2016).

Quanto à atuação dos CAPS na prevenção do suicídio foi evidenciado o acolhimento como um instrumento que favorece o atendimento humanizado e também o trabalho interdisciplinar realizado pelos profissionais. Onde foi assinalado o trabalho em equipe como uma estrutura para responder as ocorrências de risco de suicídio existentes na comunidade. Outro aspecto referido na pesquisa foi a relevância da formação ou resgate do vínculo, com o paciente e com a sua família, nas circunstâncias de crises (Heck et al., 2012). Bem como a aproximação familiar do sujeito em sofrimento colabora para desmistificar algumas crenças formadas pelo paciente (Oliveira et al., 2016).

Um ponto importante a ser debatido é que o crescente desafio diante da problemática do suicídio que os profissionais da área da saúde enfrentam também é uma consequência de fatores como a deficiência na formação profissional, pois a maioria dos centros acadêmicos não apresentam em sua grade curricular disciplinas referentes à atuação do profissional 
na prevenção do suicídio. Como também, há uma ausência de políticas públicas de educação em saúde que favoreçam a formação contínua dos profissionais. Assim, esta problemática se reflete no quotidiano da atuação dos profissionais da área da saúde e tem como uma de suas consequências a internação psiquiátrica como única forma de encaminhamento percebida pelos profissionais, devido ao desconhecimento sobre a demanda do paciente que apresenta conduta suicida, dificuldade no manejo clínico e insegurança por parte dos profissionais que compõem a equipe de saúde na prática do cuidado deste tipo de demanda (Gonçalves, Silva, \& Ferreira, 2015).

Portanto, as pesquisas são unânimes em afirmar que o paciente com comportamento suicida necessita de uma assistência que contemple mais que suas demandas físicas. Sendo importante que nessas ocasiões de crise o paciente seja escutado e acolhido. Dessa forma, é fundamental que, antes de ir para sua casa, o paciente seja avaliado e encaminhado para outros dispositivos que conseguirão dar seguimento ao tratamento em saúde mental. Pois o trabalho em rede necessita ser continuamente estimulado para que ocorra uma integração entre os vários serviços de saúde, como o hospital, unidades básicas de saúde, CAPS, dentre outros (MacChiaverni, Borges \& Oliveira, 2013; Ferreira \& Gabarra, 2014; Oliveira et al., 2016).

\section{Considerações finais}

Considerando que o trabalho objetivou revisar as possíveis intervenções da psicologia para prevenção do comportamento suicida na saúde pública, foi averiguado que esta pesquisa conseguiu alcançar esses objetivos. No primeiro momento foi possível conhecer o perfil epidemiológico do suicídio no Brasil e no mundo, que apresentou uma variação das taxas de suicídio em relação à faixa etária, ao sexo e regiões geográficas. Onde o Brasil segue a tendência mundial, em que os homens praticam mais suicídio que as mulheres. Bem como o grupo que representa maior risco de suicídio são as pessoas que estão no final da adolescência e início da vida adulta e os mais idosos.

Quanto aos principais fatores de riscos para o suicídio foi demonstrado que ocorre por vários fatores e patologias, sendo o transtorno mental o fator mais estreitamente ligado, independentemente da faixa etária. Também destacou-se a tentativa prévia de suicídio como um dos principais fatores de risco. Dessa forma, foi evidenciado que não se pode definir um fator para o suicídio e sim desencadeadores, que em alguma circunstância podem estimular o comportamento suicida.

Com o levantamento das produções científicas, foi observado que as temáticas dominantes foram atuação do psicólogo na saúde pública e suas funções no contexto do SUS, como nos CAPS e nos Hospitais Gerais. E, sobretudo, a crítica dos autores acerca da atuação da equipe de saúde ao lidarem com pacientes suicidas. Na qual foi evidenciado que isso ocorre por causa da lacuna na formação desses profissionais quanto à temática do suicídio. A deficiência de conhecimentos sobre o assunto foi descrita por grande parte das pesquisas, onde os profissionais descreveram não ter recebido um treinamento institucional para lidar com o comportamento de autoagressão.

Assim, a literatura pesquisada confirma que as tentativas de tirar a própria vida têm crescido consideravelmente no Brasil e no mundo. Sendo fundamental realizar discussões acerca das estratégias que permitam superar este problema. Pois o despreparo da equipe de saúde em não saber trabalhar frente ao comportamento suicida é verificado pela carência de discussões sobre a temática nos meios acadêmicos. Dessa forma, isso está refletindo na atuação desses profissionais. Sendo assim, o psicólogo deve ser considerado como profissional de referência tanto na assistência integral ao paciente quanto na internação hospitalar. A atuação da psicologia é de fundamental importância na atenção aos sujeitos que tentam o suicídio, porém o que preocupa é o fato dos psicólogos também não receberem treinamento, pois a sua formação acadêmica é deficiente em relação à temática, assim como nos demais cursos da área da saúde.

Em decorrência deste estudo destaca-se a importância de se realizar mais pesquisas em relação à atuação do psicólogo nos Centros de Atenção Psicossocial (CAPS), que mostrem as características dos procedimentos de trabalho, o atendimento quanto à prevenção do suicídio, bem como o papel desempenhado pelo profissional dentro desse serviço. Onde se verificou nesta pesquisa essa deficiência. Pois não foi encontrado no levantamento das publicações nenhuma pesquisa que tratasse da atuação exclusiva 
do psicólogo na prevenção do suicídio. Por isso, recomenda-se que esta prática seja acompanhada por um estudo empírico, para documentar o impacto benéfico na qualidade de vida dos pacientes que apresentam comportamentos suicidas, decorrente do trabalho de psicólogos no âmbito da saúde pública. Dessa forma, os futuros trabalhos irão colaborar para a divulgação e entendimento das várias estratégias de cuidado frente ao comportamento de autoagressão e suicídio e incrementar o progresso das políticas públicas de saúde.

Por fim, espera-se que esta revisão estimule futuras investigações com a utilização de outras técnicas e métodos de investigação científica, que explorem de uma forma mais profunda a gravidade deste problema de saúde pública. Que exige das políticas públicas de saúde, o planejamento e o desenvolvimento de ações que assegurem o apoio à vida dos usuários da rede pública de saúde (Benevides, 2005). E documentem as evidências empíricas das contribuições da atuação do psicólogo na prevenção da conduta suicida no âmbito da saúde pública.

\section{Contribuições dos autores}

Santos, M. J. S. participou da concepção, delineamento, busca e análise dos dados da pesquisa, interpretação dos resultados, redação do artigo científico. Medeiros, E. M. participou da coleta de dados da pesquisa, interpretação dos dados e redação do artigo científico.

\section{Conflitos de interesses}

Nenhum conflito financeiro, legal ou político envolvendo terceiros (governo, empresas e fundações privadas, etc.) foi declarado para nenhum aspecto do trabalho submetido (incluindo mas não limitando-se a subvenções e financiamentos, participação em conselho consultivo, desenho de estudo, preparação de manuscrito, análise estatística, etc.).

\section{Referências}

Appolinário, F. (2009). Dicionário de metodologia científica: um guia para a produção do conhecimento científico. São Paulo: Atlas.

Associação Brasileira de Psiquiatria. (2014). Diretrizes para um Modelo de Atenção Integral em Saúde Mental no Brasil. Rio de Janeiro, Brasil: ABP.
Bachmann, S. (2018). Epidemiology of suicide and the psychiatric perspective. International Journal of Environmental Research and Public Health, 15(7), 1425. doi: 10.3390/ijerph15071425

Benevides, R. (2005). A psicologia e o sistema único de saúde: quais interfaces?. Psicologia \& Sociedade, 17(2),2125. Recuperado de http://www.scielo.br/scielo. php?pid=S0102-71822005000200004\&script=sci_ abstract\&tlng=pt . doi: 10.1590/S0102$\underline{71822005000200004}$

Bertolote, J. M., \& Fleischmann, A. (2015). A global perspective in the epidemiology of suicide. Suicidologi, 7(2),6-8. doi: $10.5617 /$ suicidologi.2330

Braga, L. D. L., \& Dell'Aglio, D. D. (2013). Suicídio na adolescência: fatores de risco, depressão e gênero. Contextos Clínicos, 6(1), 2-14. doi: 10.4013/ctc.2013.61.01

Campos, L. F. L. (2008). Métodos e técnicas de pesquisa em Psicologia. (4ª ed.). Campinas, Brasil: Alínea.

Carvalho, A., Peixoto, B., Saraiva, C. B., Sampaio, D., Amaro, F., Santos, J. C., ... \& Santos, N. (2013). Plano nacional de prevenção do suicídio 2013/2017. Lisboa, Portugal: Direcção Geral da Saúde. Recuperado de https://www.dgs.pt/ documentos-e-publicacoes/plano-nacional-de-prevencaodo-suicido-20132017-pdf.aspx

Conselho Federal de Medicina. (2014). Suicídio: informando para prevenir. Brasília, Brasil: Comissão de Estudos e Prevenção de Suicídio. CFM/ABP.

Conselho Federal de Psicologia. (2013). Suicídio e os desafios para a psicologia. Brasília, Brasil: Conselho Federal de Psicologia. Recuperado de site.cfp.org.br/publicacao/suicidio-e-osdesafios-para-a-psicologia

Durkheim, É. (2014). O suicídio: estudo de sociologia. (Trad. Andréa Stahel M. da Silva). (1 ${ }^{\text {a }}$ ed.).São Paulo, Brasil: Edipro.

Ferreira, C. L. B. (2014). Trajetória do Acompanhamento em Saúde Mental dos Pacientes após o Atendimento na Unidade de Emergência por Tentativa de Suicídio. (Dissertação de Mestrado). Universidade Federal de Santa Catarina). Recuperado de https://repositorio.ufsc.br/ handle/123456789/129453

Ferreira, C. L. B., \& Gabarra, L. M. (2015). Pacientes em risco de suicídio: avaliação da ideação suicida e o atendimento psicológico. Journal of Health Sciences, 16(2), 113-122. doi: 10.17921/2447-8938.2014v16n2p\%25p 
Figueiredo, A. E. B., Silva, R. M., Mangas, R. M. N., Vieira, L. J. E. S., Furtado, H. M. J., Gutierrez, D. M. D., \& Sousa, G. S. (2012). Impacto do suicídio da pessoa idosa em suas famílias. Ciência \& Saúde Coletiva, 17(8), 1993-2002. Recuperado de https://www.scielosp.org/scielo.php?pid=S1413$81232012000800010 \&$ script=sci_abstract\&tlng=pt

Freitas, J. L., Prado, A. S., Mathias, B., Greschuck, G. R., \& Neto, J. D. (2013). Revisão bibliométrica das produções acadêmicas sobre suicídio entre 2002 e 2011. Revista Psicologia em Pesquisa, 7(2),251-260. Recuperado de http://pepsic. bvsalud.org/scielo.php?script=sci_arttext\&pid=S198212472013000200013\&lng=pt\&nrm=i\&tlng=pt. doi: 10.5327/Z1982-1247201300020013

Galvão, T. F., \& Pereira, M. G. (2014). Revisões sistemáticas da literatura: passos para sua elaboração. Epidemiologia e Serviços de Saúde, 23(1), 183-184. Recuperado de http:// scielo.iec.gov.br/scielo.php?script=sci_arttext\&pid=S167949742014000100018\&lng=en\&nrm=iso\&tlng=en. doi: $10.5123 / \mathrm{S} 1679-49742014000100018$

Gonçalves, P. I. E., Silva, R. A., \& Ferreira, L. A. (2015). Comportamento suicida: percepções e práticas de cuidado?. Psicologia Hospitalar, 13(2), 64-87. Recuperado de http://pepsic.bvsalud.org/scielo.php?script=sci_ arttext\&pid=S1677-74092015000200005\&lng=pt\&tlng=pt

Gondim, D. S. M. (2015). A Intervenção da Psicologia: Tentativas de suicídio e urgência Hospitalar. Revista Científica da Faculdade de Medicina de Campos, 10(2), 12-16. Recuperado de http://www.fmc.br/wp-content/uploads/2016/04/RevCient-FMC-2-2015-12-16-1.pdf

Heck, R. M., Kantorski, L. P., Borges, A. M., Lopes, C. V., Santos M. C., Santos, M., C. S.,... Pinho., L.B. (2012). Ação dos profissionais de um centro de atenção psicossocial diante de usuários com tentativa e risco de suicídio. Texto \& Contexto Enfermagem, 21(1), 26-33. Recuperado de http:// www.scielo.br/pdf/tce/v21n1/a03v21n1

Ferreira Júnior, A. (2015). O comportamento suicida no Brasil e no mundo. Revista Brasileira de Psicologia, 2(1), 1528. Recuperado de http://revpsi.org/wp-content/ uploads/2015/04/Ferreira-Junior-2015-O-comportamentosuicida-no-Brasil-e-no-mundo.pdf

Kohlrausch, E. R. (2012). Avaliação das ações de saúde mental relacionadas ao indivíduo com comportamento suicida na Estratégia Saúde da Família. (Tese de Doutorado). Universidade Federal do Rio Grande do Sul. Recuperado de http://hdl.handle.net/10183/69802

Koller, S. H., Couto, M. C. P. P., \& Hohendorff, J. V. (2014). Manual de produção científica. Porto Alegre, Brasil: Penso.
MacChiaverni, J., Borges, L. M., \& Oliveira, L. D. B. (2013). Instrumento para registro de atendimento psicológico a tentativas de suicídio. Barbarói, (39), 129-148. Recuperado de https://search.proquest.com/open view/04df85788cfd8da5bf000a547f3a2446/1?pqorigsite $=$ gscholar $\& \mathrm{cbl}=2029530$

Machado, D. B., \& Santos, D. N. (2015). Suicídio no Brasil, de 2000 a 2012. Jornal Brasileiro de Psiquiatria, 64(1), 45-54. doi: 10.1590/0047-2085000000056

Madeira, J. D. (2015). Intervenção com a pessoa em crise suicida aos três níveis de prevenção: Prevenção primária, secundária e terciária. (Dissertação de Mestrado). Instituto Politécnico de Setúbal). Recuperado de http://hdl.handle. net/10400.26/7874.

Marín-León, L., Oliveira, H. B., \& Botega, N. J. (2012). Suicide in Brazil, 2004- 2010: the importance of small counties. Revista Panamericana de Salud Publica, 32, 351-359. Recuperado de http://iris.paho.org/xmlui/ bitstream/handle/123456789/9245/v32n5a05_351-9. pdf?sequence=1\&isAllowed=y. doi: 10.1590/s102049892012001100005

Miranda, T. G. (2014). Autópsia psicológica: compreendendo casos de suicídio e o impacto da perda. (Dissertação de Mestrado). Universidade de Brasília, Brasília. Recuperado de http:// repositorio.unb.br/handle/10482/16392.

Oliveira, C. T., Collares, L. A., Noal, M. H. O., \& Dias, A. C. G. (2016). Percepções de uma equipe de saúde mental sobre o comportamento suicida. Gerais: Revista Interinstitucional de Psicologia, 9(1), 78-89. Recuperado de http://pepsic. bvsalud.org/scielo.php?script=sci_arttext\&pid=S1983$82202016000100007 \&$ Ing=pt\&tlng=pt

Organização Mundial de Saúde. (2012). Ação de saúde pública para a prevenção de suicídio: uma estrutura. Genebra, Suíça: OMS. Recuperado de: http://site.cfp.org.br/wp-content/ uploads/2013/07/documento-suic\%C3\%ADdio-traduzido. pdf

Organização Pan-Americana da Saúde. (2016). Prevention of suicidal behavior. Washington, DC: OPAS, 2016. Recuperado de http://iris.paho.org/xmlui/bitstream/ handle/123456789/31166/9789275119198-eng. pdf?sequence $=1$ \&isAllowed=y

Schlösser, A., Rosa, G. F. C., \& More, C., L.,O.,O. (2014). Revisão: comportamento suicida ao longo do ciclo vital. Temas em Psicologia, 22(133),133-145. Recuperado de http://pepsic. bvsalud.org/scielo.php?script=sci_arttext\&pid=S1413389X2014000100011\&lng=pt\&nrm=iso. doi: $10.9788 /$ TP2014.1-11 
Silva, B. F. A., Prates, A. A. P., Cardoso, A. A., \& Castro, N.G.M.B.R. (2018). O suicídio no Brasil contemporâneo. Sociedade e Estado, 33(2), 565-579. Recuperado de http://www.scielo. br/pdf/se/v33n2/0102-6992-se-33-02-00565.pdf. doi: $10.1590 / \mathrm{s} 0102-699220183302014$

Stefanello, B., \& Furlanetto, L. M. (2012). Ideação suicida em pacientes internados em enfermarias de clínica médica: prevalência e sintomas depressivos associados. Jornal Brasileiro de Psiquiatria, 61(1),2-7. Recuperado de http://www.scielo.br/scielo.php?pid=S0047-

$20852012000100002 \&$ script=sci_abstract\&tlng=pt. doi: 10.1590/S0047-20852012000100002

Teti, G. L., Rebok, F., Rojas, S. M., Grendas, L., \& Daray, F. M. (2014). Systematic review of risk factors for suicide and suicide attempt among psychiatric patients in Latin America and Caribbean. Revista Panamericana de Salud Pública, 36(2), 124-133. Recuperado de http://iris. paho.org/xmlui/bitstream/handle/123456789/9947/08. pdf?sequence $=1$ \&isAllowed $=y$

Trigueiro, A. (2015). Viver é a melhor opção: a prevenção do suicídio no Brasil e no mundo. ( $2^{\mathrm{a}}$ ed.). São Bernardo do Campo, Brasil: Correio Fraterno.

Vidal, C. E. L., \& Gontijo, E. D. (2013). Tentativas de suicídio e o acolhimento nos serviços de urgência: a percepção de quem tenta. Cadernos de Saúde Coletiva, 21(2),108-114. Recuperado de http://www.scielo.br/scielo.php?script=sci_ arttext\&pid=S1414-462X2013000200002\&lng=en\&nrm=i so. doi: 10.1590/S1414-462X2013000200002

Waiselfisz, J. J. (2014). Os jovens do Brasil: Mapa da violência 2014. Brasília, Brasil: Recuperado de http://www. mapadaviolencia.org.br/pdf2014/Mapa2014_JovensBrasil. pdf

Wasserman, D., Cheng, Q., \& Jiang, G. X. (2005). Global suicide rates among young peaple aged 15-19. World Psychiatry, 4(2),114-120. Recuperado de https://www.ncbi.nIm.nih. gov/pmc/articles/PMC1414751/

Zana, A. R. O., \& Kovács, M. J. (2013). O Psicólogo e o atendimento a pacientes com ideação ou tentativa de suicídio. Estudos e pesquisas em Psicologia, 13(3), 897-921. Recuperado de http://pepsic.bvsalud.org/scielo.php?script=sci_ abstract\&pid=S1808-42812013000300006 